\title{
Visualization of Topological Microstructures of Mesoporous Silicate Hollow Sphere with Confocal Microscopy
}

\author{
Chung-Yuan Mou, Te-Chen YANG ${ }^{1}$, Hong-ping LIN $^{2}$ and Fu-Jen $\mathrm{KAO}^{3 *}$ \\ Department of Chemistry, National Taiwan University, Taipei 106, Taiwan \\ ${ }^{1}$ Department of Physics, National Sun Yat-Sen University, Kaohsiung 80424, Taiwan \\ ${ }^{2}$ Department of Chemistry, National Chenkung University, Tainan 701, Taiwan \\ ${ }^{3}$ Institute of Electro-Optical Engineering, National Sun Yat-Sen University, Kaohsiung 80424, Taiwan
}

(Received September 30, 2003; accepted January 8, 2004; published April 9, 2004)

We demonstrate the use of confocal fluorescence microscopy in visualizing the exotic 3D topological structure of pillaredhollow spheres of mesoporous silicates. The mesoporous structures are labeled by rhodamine $6 \mathrm{G}$ dye to facilitate visualization. The optical sectioning capability and noninvasiveness of confocal microscopy not only further clarifies these hollow spheres' unique topological structure but also enables mapping of their genus distribution.

[DOI: 10.1143/JJAP.43.1525]

KEYWORDS: mesoporous silicate, confocal microscopy

Mesoporous aluminosilicate is one of the new materials that demonstrate formation of novel hierarchical structures with at least two length scales. ${ }^{1-3)}$ The nanopores within them are an ideal environment for the inclusion of molecular guests with different sizes, shapes, and electronic properties. They form a host-guest system that promises a tremendous number of combinations with specific properties. ${ }^{4)}$

Lin and coworkers ${ }^{2,3,5)}$ have reported that hollow mesoporous Mobil-Composite-of-Matter $(\mathrm{MCM}-41)^{6)}$ silicate microspheres of various topological genus ranks ${ }^{7)}$ can be obtained from a solution of myristyltrimethylammonium bromide $\left(\mathrm{C}_{14} \mathrm{TMAB}\right)$, butanol, and sodium silicate by careful control of the acidification rate. The calcined products consist of a high yield $(\sim 98 \%)$ of uniform-sized hollow spheres of $5.0 \pm 1.0 \mu \mathrm{m}$ diameter. The inside of the microspheres has an intricate pillar structure possessing various topological genus ranks. The walls of these microspheres consist of a hexagonal arrangement of nanochannels in latitude directions (Fig. 1). The growth mechanism of these hollow spheres was proposed as a lamellar-tohexagonal phase transformation process on a vesicle surface $^{2,3,5)}$ The center pillar is formed as a result of avoiding the high energy cost of closing the north/south pores. However, the detailed 3D mesoscopic structure within these hollow spheres is still not clearly revealed.

Confocal microscopy ${ }^{8)}$ has been demonstrated in imaging the 3D mesostructures of this class of materials. ${ }^{9)}$ It provides noninvasive optically sectioned imaging and considerably enhances the sensitivity and definition of imaging. On the other hand, the nanochannels within the mesoporous silicates exhibit strong chemical affinity to the rhodamine 6G (R6G) dye molecules. This affinity markedly facilitates labeling of the intricate structure within the hollow sphere and is the key to $3 \mathrm{D}$ visualization of the mesostructures by confocal fluorescence microscopy. Various topological genus structures and distribution of these hollow spheres can also be clearly revealed. We employ an invertedmicroscope-based laser scanning confocal setup (IX-70 + FV-500, Olympus) so that samples in liquid form can be observed using a high-numerical-aperture (NA) lens. The $488 \mathrm{~nm}$ laser line from an argon ion laser is used for exciting the R6G dye. The combination of a dichroic mirror and a

*E-mail address: fujenkao@ms21.hinet.net

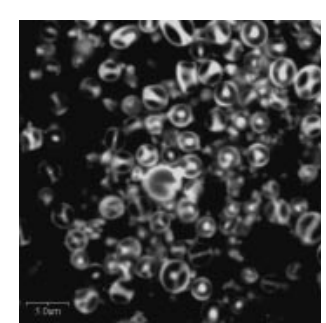

(a)

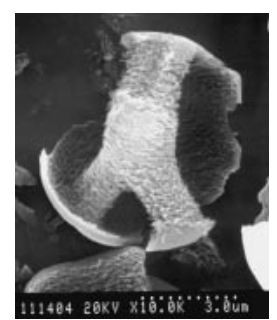

(b)

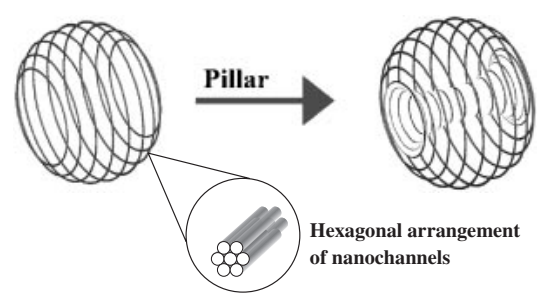

(c)
Fig. 1. (a) Fluorescence micrograph showing the morphology of the sample. (b) SEM image (c) Schematic for the formation of the pillarwithin-spheres morphology of MCM-41: Partial hexagonal nanochannels formed on the shell of spheres. The hexagonal phase grows toward poles and could not close the channels at the poles. This leads finally to the formation of the center hollow pillar to relieve the energy singularity at the poles.

band-pass filter enables effective discrimination of fluorescence from the exciting laser and other background. The acquired images are further enhanced by image processing (AutoDeblur, Autoquant) and 3D reconstruction (AutoVisualize, Autoquant) software.

To facilitate 3D fluorescence imaging, the samples are prepared by the following methods. One way is to immerse the virgin hollow spheres in the winter green oil $(n \sim 1.51)$ based R6G solution $(\sim 100 \mu \mathrm{M})$ and place them on a glass cover slide for observation. Winter green oil is used for its chemical stability as a solvent and has an index of refraction very close to that of glass. In this way, the possible spherical aberration can be minimized and the spatial resolution maximized. Additionally, autofluorescence from these spheres can also be markedly enhanced by annealing the samples in $\mathrm{N}_{2}$ environment. ${ }^{10)}$ In this case, clear winter green oil is used for the sole purpose of index matching. 


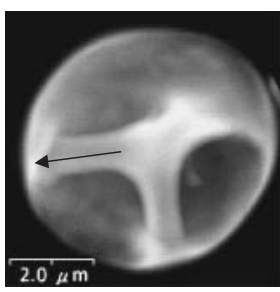

(a)

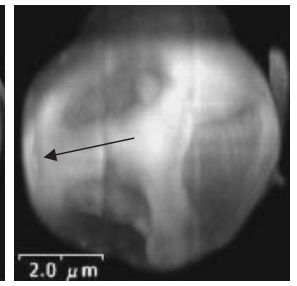

(b)

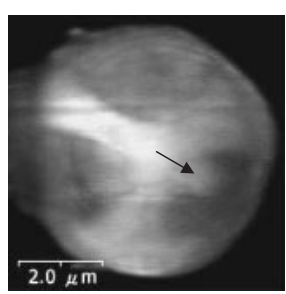

(c)

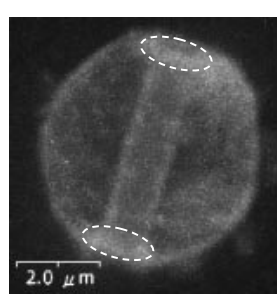

(a)

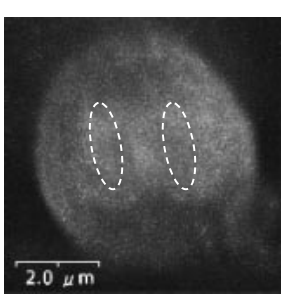

(b)

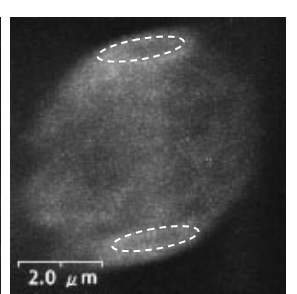

(c)

Fig. 2. The (a) $X Y$-, (b) $X Z$-, and (c) $Y Z$-sectioned views of an R6Gloaded hollow sphere. The arrows indicate the direction of the pillar as seen from the corresponding sectioned views. Note also that because of the spindle shape of the point-spread function, the spatial resolution in the $Z$-direction is not as high as in the $X Y$ plane.

With fluorescence microscopy, hollow spheres of various genus ranks can be seen [Fig. 1(a)]. All the spheres are of the following topological structures, pillar-within-sphere (PWS) (genus $=1$ ), fork-within-sphere (genus $=2)$, and crosswithin-sphere (genus = 3). ${ }^{5)}$ A broken genus 3 structure is seen in the SEM image of Fig. 1(b). Thus far, we have not observed structures such as spherical shell (genus $=0$ ), solid sphere, or structures with genus higher than 3. Structures of genus rank higher than 3 or lower than 1 are probably not energetically feasible.

Strong fluorescence is observed from the shell and the pillar structure within when the PWS spheres are immersed in the R6G solution. Figures 2(a), 2(b) and 2(c) shows the $X Y$-, $X Z$ - and $Y Z$-sectioned views, respectively, of an R6Gloaded hollow sphere (genus $=3$ ). We see that the fluorescence signal from the rim of the central pillar is stronger. Thus, the pillars are hollow to a large extent as proposed in the model in Fig. 1(c). The central pillar appears hollow, but it is also possible that excess silicates may condense inside. Three-dimensional reconstruction of the image can be performed to grasp the $2 \mathrm{D}$ rotation of the stereostructure in micron scale. They are available at the web address provided at the end of this paper. From rotation of the image, one does see the spherically symmetric thin shell and the solid central pillar.

The confocal fluorescent images of $\mathrm{N}_{2}$-annealed hollow spheres are shown in Fig. 3. It was shown that the autofluorescence is attributed to the transition in $\mathrm{Si}-\mathrm{OH}$ complexes located on the channel surface. ${ }^{10)}$ The fluorescence confocal images in Fig. 3 clearly indicate that the PWS is indeed a hollow sphere with a pillar in the center, in which the interior of the pillar gives weaker fluorescence. Again, this confirms that the central pillar is partially hollow.

The pillars (or cross) within the spheres do not necessarily form a tetrahedron, as shown in Fig. 2. The pillars' orientation $(\theta, \phi)$, with $Z$-axis pointed out of the paper, can be calculated from the cross-sectional views shown below using the spherical coordinate notation:

$$
\begin{aligned}
\cos (\phi) & =\cos \left(\theta_{x y}\right) \\
\tan (\theta) & =\tan \left(\theta_{y z}\right) / \sin \left(\theta_{x y}\right), \\
& =\frac{1}{\tan \left(\theta_{x z}\right) \cos \left(\theta_{x y}\right)}
\end{aligned}
$$

where $\theta_{x y}, \theta_{x z}$, and $\theta_{y z}$ denote angles between the pillar and the $X$-axis of $X Y, X Z$, and $Y Z$ cross-sectional views,

Fig. 3. The (d) $X Y$-, (e) $X Z$-, and (f) $Y Z$-sectioned views of an $\mathrm{N}_{2-}$ annealed hollow sphere. The images appear grainy. It is attributed to the much lower fluorescence than that of the R6G-loaded hollow spheres. The dashed circles indicate the north and south poles of the pillar to facilitate orientation identification.

respectively. Note that eq. (2) exhibits a certain redundancy in expressing the tilting angle relative to the $Z$-axis, $\theta$. It is useful, however, in double-checking the accuracy of measurements. For example, $\theta_{x y}, \theta_{x z}$, and $\theta_{y z}$ of the pillar, as marked on Fig. 2, are measured to be $188^{\circ}, 194^{\circ}$, and $330^{\circ}$, respectively. The calculated orientation angles, $\theta$ and $\phi$, are approximately $76^{\circ}$ and $188^{\circ}$, respectively. Examining many images, we conclude that the angles of the pillar of the genus 3 are distributed in a random manner. They are far from tetrahedral. This implies that the nucleation of the hexagonal phase on the shell is random and they determine the orientation of the pillars.

In summary, we have demonstrated the $3 \mathrm{D}$ visualization of mesoporous aluminosilicate hollow spheres. When compared with many other microscopic techniques, confocal microscopy is advantageous in its 3D optical sectioning capability, noninvasiveness, and high sensitivity despite its lack of spatial resolution in directly resolving nanosized structures. These capabilities are crucial in studying local dynamics and environment experienced by guest molecules that can be selectively incorporated into the porous hosting material since multiscale ordering of materials is becoming a powerful technique for the creation of macroscopic devices. $^{11)}$

We gratefully acknowledge the financial support from the National Science Council of Taiwan under grant Nos. NSC91-2112-M-110-009 and NSC91-2736-L-110-001 and from the Ministry of Education of Taiwan under grant No. 89-B-FA08-1-4.

1) H. P. Lin and C. Y. Mou: Account Chem. Research 35 (2002) 927.

2) C. Y. Mou and H. P. Lin: Pure Appl. Chem. 72 (2000) 137.

3) H. P. Lin and C. Y. Mou: Science 273 (1996) 765.

4) A. Stein, B. J. Melde and R. C. Schroden: Adv. Mater. 12 (2000) 1403

5) H. P. Lin, Y. R. Cheng and C. Y. Mou: Chem. Mater. 10 (1998) 3772.

6) C. T. Kresge, W. J. Roth, J. C. Vartuli and J. S. Beck: Nature 358 (1992) 710 .

7) S. Hildebrandt and A. Tromba: The Parsimonious Universe: Shape and Form in the Natural World (Springer-Verlag, New York, 1996).

8) C. J. R. Sheppard and D. M. Shotton: Confocal Laser Scanning Microscopy (Springer-Verlag, New York, 1997).

9) C. Seebacher, J. Rau, F.-W. Deeg, C. Bräuchle, S. Altmaier, R. Jäger and P. Behrens: Adv. Mater. 13 (2001) 1374.

10) H. J. Chang, Y. F. Chen, H. P. Lin and C. Y. Mou: Appl. Phys. Lett. 78 (2001) 3791

11) C. Sanchez, G. J. de A. A. Soler-Illia, F. Ribot, T. Lalot, C. R. Mayer and V. Cabuil: Chem. Mater. 13 (2001) 3061 